\title{
¿Cómo influye el contexto al momento de hacer repartos?'
}

\author{
How does the context when making deals?
}

Como o contexto ao fazer negócios?

Recibido: mayo de 2013

Aceptado: agosto de 2013
Mg. Eruin Alonso Sánchez Ordoñez ${ }^{2}$

\section{Resumen}

La organización de contenidos en el área de matemáticas para grado séptimo, incluye una unidad relacionada con razones, proporciones y proporcionalidad y dentro de ésta se contempla el trabajo con repartos proporcionales. Infortunadamente la naturaleza proporcional que dan los estudiantes a dichos repartos, viene inducida por el profesor quien de antemano ha seleccionado problemas típicos que aparecen en los libros de texto, para los cuales se espera que el estudiante haga un reparto proporcional directo o inverso. Por tanto, es interesante proponer a los estudiantes situaciones previas a la instrucción formal para determinar qué tipo de repartos predominan y cuál es la influencia del contexto de la situación y del estudiante al decidir entre repartos proporcionales y repartos equitativos.

Palabras clave: Matemáticas escolares, repartos; relaciones numéricas; razón y proporcionalidad; equidad; contexto.

\begin{abstract}
BThe content organization in the area of math for seventh grade, includes a related unit ratios, proportions and proportionality and within this work is contemplated proportional distributions. Unfortunately the proportional nature that give students such distributions, is induced by the teacher who selected beforehand typical problems that appear in textbooks, for which the student is expected to make a direct or inverse proportional distribution. It is therefore interesting to propose situations students prior to formal instruction to determine what kind of deals predominate and what is the influence of the context of the situation and the student to decide between distributions proportional and equitable distributions.
\end{abstract}

Keywords: school Mathematics, deals, numerical relationships, reason and proportionality, fairness, context.

\section{Resumo}

A organização dos conteúdos da área de matemática para sétima série, inclui uma unidade de rácios relacionados, proporções e proporcionalidade

1 Artículo de Investigación

2 Institución Educativa “Los Comuneros” Popayán, Colombia. Contacto: eruinalonso@hotmail.com 
e dentro deste trabalho é contemplado distribuições proporcionais. Infelizmente, a natureza proporcional que dar aos alunos tais distribuições, é induzida pelo professor que selecionou os problemas típicos de antemão que aparecem nos livros didáticos, para o qual se espera que o aluno faça uma distribuição proporcional direta ou inversa. Por isso, é interessante propor aos alunos situações antes de instrução formal para determinar que tipo de negócios predominam e qual é a influência do contexto da situação e do aluno para decidir entre as distribuições distribuição proporcional e equitativa.

Palavras-chave: Matemática da escola, negócios, relações numéricas, a razão ea proporcionalidade, da justiça, do contexto.

\section{Introducción}

En el desarrollo del proyecto de investigación Razones, Proporciones y Proporcionalidad en términos de variación y correlación entre magnitudes: Una posible forma para comprender la construcción de dichos objetos matemáticos ${ }^{3}$, se aplicó una situación de reparto proporcional para determinar las estrategias previas a la instrucción, desplegadas por los estudiantes para resolver dicha situación ${ }^{4}$. Luego de analizar diferentes procedimientos escritos, empleados por los estudiantes, y respuestas verbales dadas a ciertas preguntas puntuales sobre dichos procedimientos, se observó que podría seguirse indagando y profundizando en los sistemas de prácticas determinados en este análisis. De esta forma se decidió volver a implementar la misma situación, tres años después, con un grupo de estudiantes distinto pero de la misma institución. El proceso en este nuevo análisis fue aplicar una situación previa sustentada en las relaciones de amistad y compañerismo en la cual también estaban involucrados los repartos y mayoritariamente inclinada a la realización de análisis cuantitativos no numéricos, a continuación se aplicó la situación del proyecto de investigación ya mencionado y posteriormente una situación similar a ésta última, cambiando datos numéricos y el contexto de aplicación. Finalmente se puso a los estudiantes en una situación real .

La idea es determinar en qué contextos predominan los repartos equitativos por encima de los proporcionales y si el tipo de reparto (equitativo, proporcional) depende de variables como la edad y el entorno en el que se desenvuelve el estudiante, así como si estos repartos cambian cuando se pasa de una situación hipotética a una situación concreta real. $^{5}$

\section{Marco teórico}

Teoría Antropológica de lo Didáctico. Para Bosh y Chevallard (1999) el estudio del conocimiento matemático como un conjunto de prácticas sociales institucionalizadas requiere de una forma de análisis que permita la descripción y el estudio de las condiciones de su realización. Dicho análisis, es lo que desde la TAD se ha denominado organización matemática $(\mathrm{OM})$ o praxeología, o en palabras de Espinoza y Azcárate (2000) una OM permite modelizar el conocimiento matemático como actividad humana.

Estas praxeologías, propuestas por el enfoque antropológico, están compuestas de tipos de

3 Este proyecto se desarrolló como requisito para optar al título de Magister en Educación. Línea enseñanza de las Ciencias y la tecnología de la Universidad del Cauca y tuvo como objetivo identificar los sistemas de prácticas desplegados por los estudiantes en la solución de situaciones de variación y de cambio e identificar de qué manera esos sistemas dan forma a los conceptos de razón, proporción y proporcionalidad.

4 Los alcances en la aplicación de esta situación pueden ser vistos en Sánchez (2013).

5 Las anteriores situaciones eran hipotéticas en cambió esta cuarta se realizó con material real, dinero de verdad que quedó en manos de los estudiantes. 
situaciones (S), problemas y de técnicas, las cuales constituyen la praxis o conocimientos técnicos y de tecnologías y teorías que constituirán el logos o saber. Según Espinoza y Azcárate (2000) las técnicas se entienden como ciertas maneras de hacer, esto es, como procedimientos que pueden ser empleados para resolver los problemas; las tecnologías como los discursos que sustentan, describen, explican y justifican los procesos matemáticos que ahí se encuentran involucrados y los cuales se espera sean más adelante institucionalizados en los procesos de enseñanza y de aprendizaje y la teoría como el argumento formal que permite justificar rigurosamente dicha tecnología.

De lo anterior se puede determinar que los objetos de conocimiento matemático surgen de prácticas con las matemáticas ubicadas en diversos contextos geográficos y culturales, en tal sentido, D’Amore y Godino (2007); Godino, Batanero y Font (2008), entienden una práctica matemática como una actuación particular, o conjunto de actuaciones, en el abordaje de problemas matemáticos específicos (de un individuo o de una institución). Esta práctica está determinada por formas de razonar, comunicar, validar o generalizar y habitualmente no existe de manera aislada sino que está asociada a sistemas de prácticas que interaccionan entre sí.

Educación Matemática Crítica. Surge a partir de los postulados de la Educación Crítica. Para Skovsmose (1999) la crítica y la Educación deben ir ligadas, pues de lo contrario, la Educación correría el riesgo de convertirse tan solo en una entrega de información o en una socializadora de la juventud dentro de la cultura existente, además son las instituciones educativas las encargadas de reaccionar frente a la naturaleza crítica de la sociedad. En este sentido, si las prácticas y la investigación educativa abordan los conflictos y las crisis de la sociedad y revelan las desigualdades y la represión de cualquier tipo, se está en presencia de una Educación Crítica. Se entiende crítica desde la perspectiva Hegeliana, es decir, como aquella que trata de aclarar las condiciones políticas y económicas del desarrollo de las ideas.

La Educación crítica debe ser una reacción a todo tipo de característica crítica de la sociedad. Tiene que tomar en cuenta todos los tipos de desigualdades (clase, raza, credo, nivel socioeconómico, género, edad, etc.). Una Educación crítica debe tener en cuenta el contexto crítico de la escolaridad y tratar de desarrollar posibilidades para crear una conciencia acerca de los conflictos, al igual que proporcionar las competencias que sean importantes para manejar tales situaciones críticas.

En la Educación Crítica se entiende la alfabetización como el proceso que permite desarrollar condiciones para que los seres humanos se ubiquen en la historia y reconozcan su posición en la sociedad para poder funcionar en ella. A partir de ello, las personas dejan de ser observadores para convertirse en actores, en este orden de ideas la alfabetización tiene como objetivo no solo mejorar la comprensión de la sociedad sino también contribuir a su transformación. Particularmente en la alfabetización matemática, se debe tener en cuenta que aunque inicialmente este término denominaba la habilidad para calcular y usar técnicas formales y matemáticas, actualmente se está convirtiendo en una condición previa para la emancipación social y cultural. Esta segunda concepción de alfabetización matemática es la que permite hablar de una educación matemática crítica.

Desde la perspectiva de la Educación Matemática crítica las matemáticas pueden adquirir no solo un carácter interpretativo sino también formativo. En este sentido, las matemáticas producen nuevas invenciones de la realidad ya que colonizan gran parte de ésta y la reorganizan. Por otro lado las matemáticas dan forma a la sociedad, lo cual muestra a las matemáticas como un agente de desarrollo social.

Razonamiento proporcional. Según Lamon (2007) este tipo de razonamiento tiene que ver con suministrar argumentos que permitan soportar las enunciaciones que se hacen con respecto a las relaciones estructurales entre cuatro cantidades. Estas enunciaciones están hechas en contextos que al mismo tiempo involucren la covariación entre cantidades y la invariancia de razones o productos. Por tanto el razonamiento proporcional podría ser considerado como aquella habilidad que permite no sólo diferenciar la relación multiplicativa entre 
dos cantidades sino también la capacidad de poder extender dicha relación a otro par de cantidades.

Metodología del taller

La idea es poner a los participantes en situación, por tal razón se los invitará a trabajar aproximadamente de la misma forma como lo hicieron los estudiantes. Tal desarrollo se hará a partir de tres momentos:

- Primer momento: Proponer a los participantes que resuelvan las tres situaciones aplicadas a los estudiantes.

- Segundo momento: Realizar una presentación formal de los elementos teóricos que sustentan el taller.

- Tercer momento: Presentar a los participantes los resultados obtenidos en la implementación a los estudiantes de grado séptimo.

- Cuarto momento: Discusión a partir de la presentación de resultados hecha en el tercer momento y de los resultados obtenidos en el primer momento.

Se anexan las situaciones.

\section{Referencias}

Bosch, M., \& Chevallard, Y. (1999). Ostensifs et sensibilité aux ostensifs dans l'activité mathématique. Recherches en didactique des mathématiques 19 (1), 77-124.
D’amore, B., \& Godino, J. (2007). El enfoque ontosemiótico como un desarrollo de la teoría antropológica en didáctica de las matemáticas. Revista Latinoamenricana de Investigación en Matemática Educativa, 10 (2), 191-218.

Espinoza, L., \& Azcarate, C. (2000). Organizaciones matemáticas y didácticas en torno al objeto "limite de una función": una propuesta metodológica para el análisis. Enseñanza de las Ciencias, 18 (3), 355-368.

Godino, J., Batanero, C., \& Font, V. (2008). Un enfoque ontosemiótico del conocimiento matemático. Consultado en diciembre 15, 2009. Disponible en http://www.ugr.es/local/jgodino/indice_eos.htm

Sánchez, Eruin. (2013). Razones, proporciones y proporcionalidaden una situación de reparto: una mirada desde la teoría antropológica de lo didáctico. Revista Latinoamericana de Investigación en Matemática Educativa, 16 (1), 65 - 97.

Skosmovse, Ole. (1999). Hacia una filosofía de la Educación Matemática Crítica. Bogotá: Una Empresa Docente. 


\section{ANEXO 1}

\section{Situación 2: Repartamos el premio}

Luisa, Pedro, José y Martha (quienes no se conocen) compraron una boleta para la rifa de 12'000.000 de pesos en efectivo. El valor total de la boleta es $\$ 10.000$. Para la compra Luisa aportó 1.000 pesos, Pedro 2.000, José 3.000 y Martha 4.000 pesos.

\section{(Recuerda escribir las operaciones que realizas)}

1. Si las cuatro personas se ganan la rifa :

a. ¿Quién recibe más dinero? ¿Por qué?

b. ¿Quién recibe menos dinero? ¿Por qué?

c. ¿Qué cantidad de dinero le corresponde a cada uno?

2. ¿Cuánto debería recibir José si su aporte hubiese sido de 5.000 pesos?

3. ¿Cuánto debería aportar una de las tres personas si hubiera querido ganarse 9'000.000?

\section{Situación 2: Repartamos el premio}

Luisa, Pedro, José y Martha (quienes no se conocen) compraron una boleta para la rifa de 12’000.000 de pesos en efectivo. El valor total de la boleta es \$10.000. Para la compra Luisa aportó 1.000 pesos, Pedro 2.000, José 3.000 y Martha 4.000 pesos.

\section{(Recuerda escribir las operaciones que realizas)}

1. Si las cuatro personas se ganan la rifa :

a. ¿Quién recibe más dinero? ¿Por qué?

b. ¿Quién recibe menos dinero? ¿Por qué?

c. ¿Qué cantidad de dinero le corresponde a cada uno?

2. ¿Cuánto debería recibir José si su aporte hubiese sido de 5.000 pesos?

3. ¿Cuánto debería aportar una de las tres personas si hubiera querido ganarse 9'000.000?

\section{Situación 3: ¿De cuánto es mi bonificación?}

Una empresa ha otorgado una bonificación de $60^{\prime} 000.000$ de pesos en efectivo a cuatro de sus trabajadores por terminar en tiempo record un trabajo adicional. Andrés destinó 4 horas, Beatriz 6 horas, Camila 3 horas y Daniel 2 horas para ejecutar el trabajo.

\section{(Recuerda escribir las operaciones que realizas)}

1. De acuerdo con la información anterior:

a. ¿Cuál empleado recibe más dinero? ¿Por qué?

b. ¿Cuál empleado recibe menos dinero? ¿Por qué?

c. ¿Qué cantidad de dinero le corresponde a cada uno?

2. ¿Cuántas horas debería haber trabajado Camila para recibir 20’000.000 de pesos de bonificación?

3. ¿De cuánto sería la bonificación de una persona que trabajó 10 horas?

Situación 4: ¿Cuánto merezco por mi trabajo?

A un grupo de cuatro estudiantes se les entregó 4 juegos de piezas de tangram para armar 15 figuras y se les dijo que se repartirían \$ 6000 entre los cuatro de acuerdo al número de figuras completas que armaran.

Fuente: Elaboración propia 